\title{
Determining the 3D Structure of the Corona Using Vertical Height Constraints on Observed Active Region Loops
}

\author{
G. Allen Gary ${ }^{1}$. Qiang $\mathbf{H u}^{2}$. \\ Jong Kwan Lee ${ }^{3}$. Markus J. Aschwanden ${ }^{4}$
}

\begin{abstract}
The corona associated with an active region is structured by hightemperature, magnetically-dominated closed and open loops. The projected $2 \mathrm{D}$ geometry of these loops is captured in EUV filtergrams. In this study using SDO/AIA $171 \AA$ filtergrams, we expand our previous method to derive the 3D structure of these loops, independent of heliostereoscopy. We employ an automated loop recognition scheme (Occult-2) and fit the extracted loops with 2D cubic Bézier splines. Utilizing SDO HMI magnetograms, we extrapolate the magnetic field to obtain simple field models within a rectangular cuboid. Using these models, we minimize the misalignment angle with respect to Bézier control points to extend the splines to 3D (Gary, Hu, and Lee, 2013). The derived Bézier control points give the $3 \mathrm{D}$ structure of the fitted loops. We demonstrate the process by deriving the position of $3 \mathrm{D}$ coronal loops in three active regions (AR 11117, AR 11158, and AR 11283). The numerical minimization process converges and produces $3 \mathrm{D}$ curves which are consistent with the height of the loop structures when the active region is seen on the limb. From this we conclude that the method can be important in both determining estimates of the 3D magnetic field structure and determining the best magnetic model among competing advanced magnetohydrodynamics or force-free-magnetic-field computer simulations.
\end{abstract}

\footnotetext{
1 Center for Space Plasma and Aeronomic Research, The University of Alabama in Huntsville, Huntsville, AL 35899, USA email: gag0002@uah.edu

2 Center for Space Plasma and Aeronomic Research and Department of Space Science, The University of Alabama in Huntsville, Huntsville, AL 35899, USA email: qh0001@uah.edu

${ }^{3}$ Department of Computer Science, Bowling Green State University, Bowling Green, OH 43403, USA email: leej@bgsu.edu

4 Solar and Astrophysics Laboratory, Lockheed Martin

Advanced Technology Center, Dept. ADBS, Bldg. 252, 3251

Hanover St., Palo Alto, CA 90304, USA email:

aschwanden@Imsal.com
} 
Keywords: Magnetic fields, Models; Magnetic fields, Corona; Active Regions, Magnetic Fields; Chromosphere, Models; Instrumentation and Data Management

\section{Introduction}

A major objective of solar physics is to determine the three-dimensional nonpotential magnetic field in the corona that will provide critical information on the development and release of energy in major solar eruptions (e.g. flares and coronal mass ejections) and the origin of solar energetic particles (SEPs). Solar studies indicate that the coronal magnetic field plays a central role in the physics of these events (Aschwanden, 2004). However, a major solar physics problem is not being able to easily determine the magnetic field in the corona above an on-disk active region. This problem, in part, is due to the uncertainty of the height of the EUV or radio emission. Our research addresses this problem by providing an estimate of the 3D structure of the magnetic field given an appropriate magnetic-field model and by providing a numerical criteria on how well the model determines the solar magnetic field. The process builds on a recent study of Gary, Hu, and Lee (2013), Paper I, where we demonstrated the process of extending the two-dimensional (2D) coronal loop fits, as observed in extreme ultraviolet (EUV) filtergrams, into the three-dimensional (3D) coronal space.

Using appropriate magnetic-field models, our approach in Paper I was to determine the optimum magnetic-field model by employing coronal imagery and extending the traced loops into a 3D coronal space. The important points of the first paper on using cubic Bézier splines included a rapid and flexible manual method to map on-disk coronal loops of a 2D EUV image into a 3D coronal loop. In this second paper, we employ the automated Occult-2 loop recognition routine (Aschwanden, 2013b). The method of Paper I employed 2D cubic Bézier splines to approximate a traced coronal loop trajectory in the plane-of-sky projection, using four control points of functions of $x$ and $y$; the third coordinate, $z$, perpendicular to the plane of the sky, is initially zero, as does this paper. However, for an active region in Paper I, the set of 2D splines, as defined in the plane of the sky by a set four control points was transformed to the best 3D splines for a particular coronal magnetic-field model using only the two central control points where the third coordinate $z$, for these two control points, is allowed to be non-zero. The location of the two end control points were assumed to be on the photosphere, with $z=0$, and their location were estimated by associating them with a photospheric foot-points which had enhanced heating via surrogates (e.g. small magnetic islands or EUV enhancements). In this paper, all four cubic Bézier control points have variable heights $(z>0)$, which are to be determined, (see Figure 1 for coordinate definitions). Paper I showed how the method could restrict the magnetic-field models derived from extrapolations of magnetograms to those admissible and inadmissible via a fitness parameter. Here we extend this concept of using the method to determine the fitness of magnetic-field models. We outline explicitly how the traced coronal loops can be 
Table 1. The time and position of the three active regions used in the study. In all cases the field of view (FOV) is in arcseconds; however for each case the FOV in HMI pixels was $700 \times 500$. The observation time of the AIA 171 filtergram and the HMI magnetogram are given in Universal Time (UT). The heliocentric latitude and longitude [lat, long] from the center of the FOV and heliocentric latitude of the center point of the disk [B0] are in degrees, with the longitude measured from the central meridian. The date and time of the limb observations of the active region are also given.

\begin{tabular}{cccc}
\hline Active Region & AR 11117 & AR 11158 & AR 11283 \\
\hline On-Disk Date & 25 October 2010 & 13 February 2011 & 6 September 2011 \\
AIA Obs. Time & $21: 00: 48.34$ & $23: 39: 37.34$ & $23: 28: 12.34$ \\
HMI Obs. Time & $20: 58: 15.50$ & $22: 34: 12.10$ & $23: 22: 22.40$ \\
Lat,Long,B0 & $-0.2,20.1,5.0$ & $-2.3,-19.6,-6.8$ & $14.0,15.1,7.3$ \\
FOV & $353.0 \times 252.1$ & $353.0 \times 252.1$ & $353.0 \times 252.2$ \\
Limb Date & 31 October 2010 & 19 February 2011 & 11 September 2011 \\
AIA Obs. Time & $23: 36: 24.24$ & $22: 35: 36.34$ & $23: 28: 24.34$ \\
\hline
\end{tabular}

employed in constraining competing magnetic-field models using the transformed 3D (coronal) splines using all four of the cubic Bézier control points. The method again uses the minimization of the misalignment angles between the magnetic field direction given by the model and the tangents of the best set of 3D field lines that match observed EUV coronal loops.

The accomplishments of this current study includes the use of the Occult-2 and the 3D Bézier extension with both the two floating (i.e. $z>0$ ) end points and the two floating intermediate control points. In particular the Occult-2 loop recognition scheme is extended by fitting its loop curves with a 2D cubic Bézier spline for each loop. The 3D vertical extension of the Bézier control points, which have all four control points floating with $z>0$, is shown to numerically converge. We employ the use of a potential-magnetic-field solution to explicitly evaluate the Occult-2 results and the 3D extension process. We use both potential and linear force-free (LFFF) magnetic fields to determine the limitations and constraints of the general method.

We use three active regions and limb comparison results to further test the process. These multiple active-region comparisons provide important estimations of the heights of the coronal loops, and hence the structure of the corona, albeit with a potential magnetic-field model.

The organization of this paper is the following: The methodology of the analysis is explained followed by application of the process to known solutions. The process is applied to three different active regions. The coronal-loop heights are obtained by using a minimum of the misalignment angle, and the resulting structure of the active region is compared to limb observations that are at a later time. 


\section{Methodology of finding the coronal structure}

The methodology of the investigation has eight main elements. The process accomplishes the following elements in order:

i) extracts a set of loops from EUV imagery using an automated loop recognition program,

ii) identifies and removes any false-positive detected loops,

iii) fits these 2D loops with a cubic Bézier spline to permit straightforward loop extension to 3D space,

iv) generates a magnetic-field solution by extrapolation of photospheric magnetograms,

v) determines the 3D heights of the identified loops using the magnetic-field model and misalignment angles,

vi) obtains an EUV limb view of the active region for comparison with the derived height structure,

vii) analyzes the derived 3D-loop structures in terms of distributions of coronalloop location and fitness with the magnetic-field model, and

viii) analyzes the implication of the structure in terms of the appropriateness of the magnetic model employed.

This study uses an effective pattern recognition code that extracts the curvilinear loops from 2D imagery. The code, called Oriented Coronal CUrved Loop Tracing-2 (Occult-2), has been recently developed by Aschwanden and associates (Aschwanden, 2013b; Aschwanden, 2010). The code is extremely fast and we employed it, rather than the semi-manual method of Gary, Hu, and Lee (2013), for its speed and and its availability under the SolarSoftWare [SSW] library. The program is written in Exelis Visual Information Solutions' Interactive Data Language [IDL]. We have employed SSW programs for the analysis presented here and we will make the programs publicly available. Aschwanden's Occult-2 loop recognition program used is the Looptracing_auto2.pro version. The selection of the appropriate tracing parameters is critical to the loop detection process. A manual iteration process is required to select the parameters that give a useful set of extracted loops, although the sets used this study are not optimized. The elements of the Occult-2 pattern recognition code are based on an intensity ridge tracing process. An image-base leveling reduces the effect of unwanted weak secondary structures. The fine loop structures are enhanced by bandpass spatial filtering. The analysis program establishes a noise threshold by using a small, programmer-selected sub-region without loops. The program is initiated with a selection of starting points from intensity maxima. Then the program determines an intensity ridge and its local direction. It performs bidirectional tracing along the ridge limited, in part, by a local curvature radius which constrains noise effects and allows minor gap bridging. After each loop is traced and recorded, a loop-erasing procedure removes the traced loop from the image and the procedure is repeated again until an ending criteria terminates 
the iterations. There are some false-positive detections in the Occult-2 process and some non-optimum detections due to overlapping loops and extra end-point curvature as a result of image noise. There are a few rare cases where two separate coronal loops were traced as a single curve. We have added a manual method of removing ostensibly false-positive detections. This rejection process only slightly reduces the total loops acquired. The overall Occult- 2 tracing process provides over 100 coronal loop segments per active region.

Two data sets are employed from Solar Dynamics Observatory (SDO). One data set is the $171 \AA$ filtergrams from the extreme ultraviolet (EUV) multiplewavelength spectroheliograph, Atmospheric Imaging Assembly (AIA). The other data set is the vector magnetograms from the Helioseismic and Magnetic Imager (HMI) (Lemen et al., 2012; Scherrer et al., 2012). The Flexible Image Transport System (FITS) files for these instruments were obtained from the Joint Science Operation Center (JSOC, Stanford University) as full disk data. The FITS files of Solar Dynamics observatory AIA $171 \AA$ filtergrams and HMI magnetic field observations (field, azimuth, inclination) were obtained for the three active regions of Table 1 (using SSW IDL read_sdo.pro). All three active regions were near the central meridian and hence a perpendicular planar geometry was assumed, which removed some of the complexity of the analysis. The HMI vector magnetic field data was computed by the HMI team from Stokes parameters derived from 36 filtergrams with weighted averages computed at 720 s intervals and using an Milne-Eddington inversion (i.e. assuming a linear source function with optical depth and absorption matrix independent of optical depth) to determine the magnetic field parameters of total field strength, inclination angle relative to the line of sight, and 180-degree azimuth. From the vector magnetic field data, the derived line-of-sight (LOS) magnetic fields are used for the magnetic models used in this paper. The high-spatial-resolution AIA $171 \AA$ filtergrams originated from the primary ion Fe IX from plasma with a characteristic temperature of $10^{5.8} \mathrm{~K}$ giving detailed images of the magnetically-confined coronal loops (Aschwanden, 2004). Limb observation employing AIA $171 \AA$ observation several days later are used for comparison (see Table 1 for the observation times). These limb observations provide an image of typical heights of the $171 \AA$ loops. The activeregion limb arrival time can be easily determined by using SSW IDL routine rot_xy.pro.

The use of the 3D cubic Bézier spline as an extension of the 2D spline was employed in Paper I to develop a numerical robustness parameter for evaluating model fitting and was applied to three different magnetic-field models to determine the optimum fit of the 3D splines. The paper had the general, finite 2D Bézier curve of degree $n=3$ (cubic) in the form:

$$
\mathbf{X}(x, y)=f(u)=\sum_{i=0}^{n} \mathbf{P}_{i+1} \beta_{n, i}(u),
$$

where $u \in[0,1]$ is a tracing parameter along the curve and $\beta_{n, i}$ is the $n^{\text {th }}$-degree polynomial basis function, the Bernstein polynomials,

$$
\beta_{n, i}(u)=\frac{n !}{i !(n-i) !} u^{i}(1-u)^{n-i}, \quad n=3,
$$




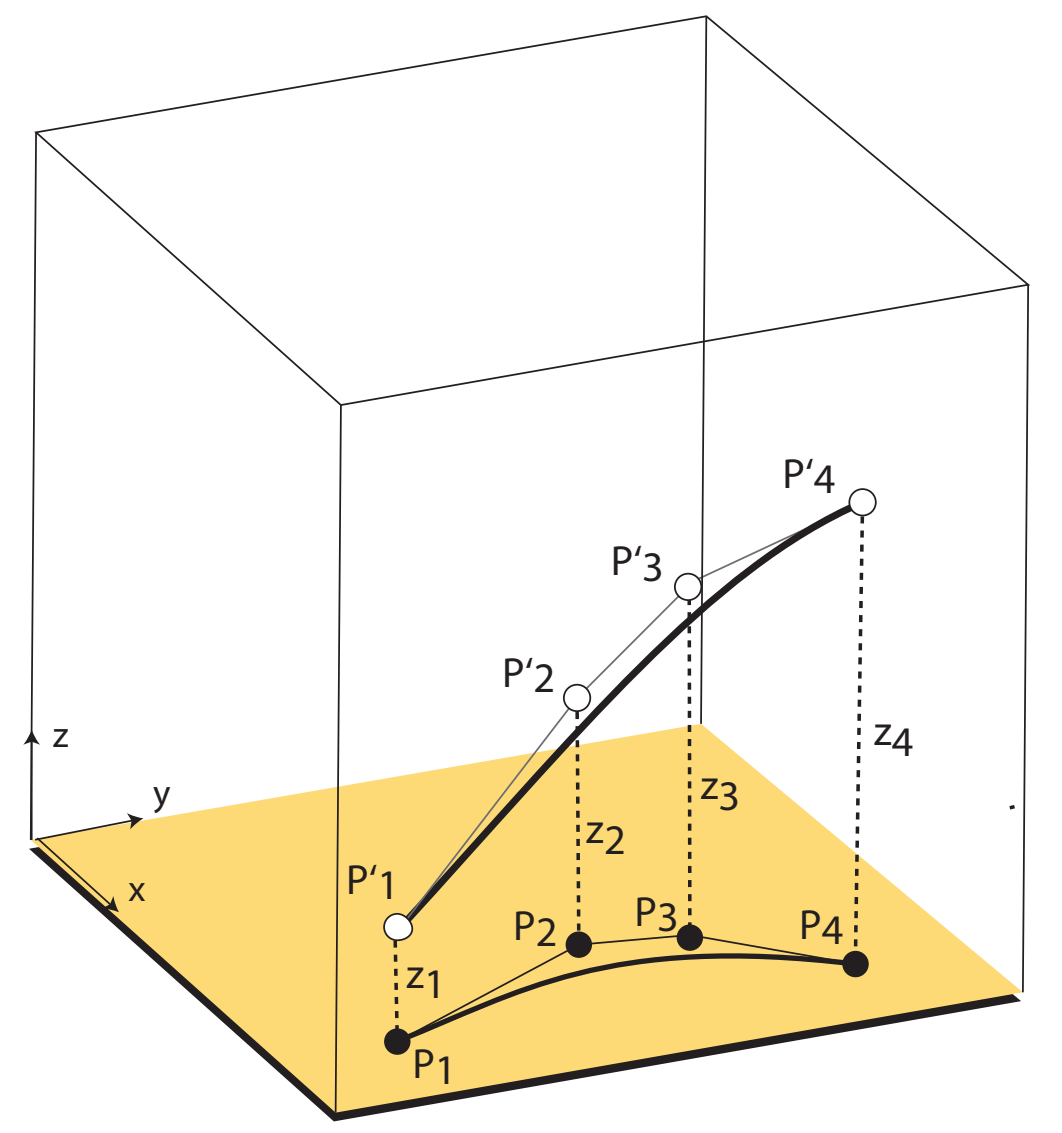

Figure 1. The cubic Bézier connection between the 3D and 2D projections. A 2D cubic Bézier curve, $\mathbf{X}(x, y)$, in the $z=0$ plane (lower thick line) is defined by the control points $\mathbf{P}_{i}\left(x_{i}, y_{i}\right), i \in(1,4)$. If the four control points (solid black circles) become 3D vectors, via $\left(x_{i}, y_{i}, 0\right)$ and if we define $\mathbf{P}_{i}^{\prime}$ as $\mathbf{P}_{i}^{\prime}\left(x_{i}, y_{i}, z_{i}\right)$, then we define the $3 \mathrm{D}$ cubic Bézier curve, $\mathbf{R}(x, y, z)$, (upper thick line) by the introduction of four parameters: $z_{1}, z_{2}, z_{3}$,and $z_{4}$. This allows the 3D-cubic Bézier spline to be non-planar. However, the two curves are related by having the same projected points lying in the image plane.

and $\mathbf{P}_{\mathbf{j}}(j=1,2,3,4)$ are position vectors $\left(x_{j}, y_{j}\right)$ called control points and are the vector parameters that control the shape of the curve (Mortenson, 1997, Equation (4.2)) Explicitly translating Equation (1) into the 3D form, the 3D cubic Bézier spline has the form:

$$
\begin{array}{r}
\mathbf{R}(x, y, z)=\mathbf{F}(u)=\left[\left[(1-u)^{3} x_{1}+3 u(1-u)^{2} x_{2}+3 u^{2}(1-u) x_{3}+u^{3} x_{4}\right],\right. \\
{\left[(1-u)^{3} y_{1}+3 u(1-u)^{2} y_{2}+3 u^{2}(1-u) y_{3}+u^{3} y_{4}\right],} \\
\left.\left[(1-u)^{3} z_{1}+3 u(1-u)^{2} z_{2}+3 u^{2}(1-u) z_{3}+u^{3} z_{4}\right]\right],
\end{array}
$$

where $\left(x_{j}, y_{j}\right.$, and $\left.z_{j}\right)$ are the coordinates of the four points, $j=1,2,3$, and 4 . The importance of the $3 \mathrm{D}$ form is that the $\mathbf{X}(x, y)$ curve is the $2 \mathrm{D}$ projection 
of the 3D curve $\mathbf{R}(x, y, z)$ onto the $z=0$ plane, for any value of the control point z-components (see Figure 1). Hence, for a coronal loop, if the four $z_{i}$ 's can be determined such that the 3D loop segments correspond to a section of the magnetic-field line then the 3D location of the coronal loop is determined from its $2 \mathrm{D}$ projection $\mathbf{X}(x, y)$. And in fact, if we have three adjacent noncoplanar loops, close enough such that the conservation of flux can be numerical employed, and one of the end points is near the photosphere, then the magnetic-field strength could be estimated along the center of these three observed loops (a flux tube) giving both the magnetic field strength and its 3D structure. Although, the loopheight locations are restrained by a given magnetic field model, the overall flux tube structure and magnetic field values are not and hence this procedure would be an additional constraint to improve magnetic modeling.

To quantify the use of the cubic Bézier curve over higher-degree curves, Paper I used a minimization process to compare the differences between the cubic curves and a series of magnetic-field lines generated from a known magnetic field configuration. Generally, a cubic Bézier spline sufficiently fits the Occult-2 tracings to within the half-width of the $171 \AA$ coronal loop.

This ability to extend the cubic Bézier splines to 3D is the reason that the Occult-2 traced loops are initially transformed into 2D cubic Bézier splines. Having the projected loops, we want to develop a method to determine the values of the four $z_{i}$ 's. This process extends the work of Paper I, where only two of the four $z_{i}$ 's were varied since the end points of the loops were assumed to be at the photospheric foot points $\left(z_{i}=0\right)$. In this paper we expand the process to all four control points and show that the process usually converges to a numerical solution. The few non-convergent cases are the result of errors in loop identification, or a particular geometry of the projected field lines (e.g. short or straight).

For the magnetic-field models, we use a set of magnetic dipoles positioned below a planar photosphere. For the collection $(N)$ of fixed-depth multi-magnetic dipole fields the 2D locations $\left(\mathbf{r}_{i 0}\right)$ and magnetic dipole strengths $\left(M_{i}\right)$ are determined using a numerical nonlinear-Powell local-minimum routine on the root-mean-squared (RMS) difference of the vertical-component of the magnetic dipole fields and the Gaussian-smoothed magnetograms. Both potential and LFFF magnetic dipoles with constant $\alpha$ are employed in the study as defined by

$$
\begin{aligned}
\boldsymbol{B}_{\text {theo }}(\mathbf{R}) & =\sum_{i=1}^{N} \boldsymbol{B}\left(\mathbf{R}, \mathbf{r}_{i 0}, M_{i}, \alpha\right) \\
& =\sum_{i=1}^{N} \nabla \times \nabla \mathbf{e}_{z} U\left(\left|\mathbf{R}-\mathbf{r}_{i 0}\right|\right)+\alpha \nabla \times \mathbf{e}_{z} U\left(\left|\mathbf{R}-\mathbf{r}_{i 0}\right|\right),
\end{aligned}
$$

where $\mathbf{e}_{z}$ is the vertical unit vector, $U(r)=\frac{M_{i} \cos (\alpha r)}{r}$ and the constant $\alpha$ parameter is zero for the potential case, (e.g. Equation 37, (Gary, 1989); Equation 3.26, (Marsh, 1996)). The Gaussian spatial smoothing of the line-of-sight field, $B_{\text {smooth }}$, gives a magnetogram at an equivalent height of about 5 arcsec into the corona, which reduces the number and better establishes the location of the important magnetic field extrema. The number and locations of the dipoles 
are determined by the set of the largest extrema values ( $\geq 250$ Gauss), and, hence, is dependent on the number of major magnetic islands of the active region. All dipoles have a depth set at $15 \mathrm{Mm}$ to have half-widths of field values approximating the magnetic field islands at $z=0$. The dipole strengths are then determined by the minimum RMS difference of $\left|B_{\text {smooth }}-B_{z}\right|$ at $z=0$, where $B_{z}$ is the z-component of $B_{\text {theo }}$ (Equation 4 ). In this paper, the LFFFmultidipole models are used to avoid, in part, of having periodic solutions as with the fast Fourier transform methods, since some of both the open and closed EUV loops reach high into the corona and the modeling of these large structures would be adversely effected by periodic boundaries. Furthermore, since the LFFFmultidipole models have analytic solutions, the calculations for the magnetic field directions avoid grid interpolations. Once the multidipole parameters are defined, the magnetic field calculations can be rapidly determined without employing any numerical grid calculations. In this paper, the multidipole-magnetic-field model is employed, however, in general, the procedure can use any magnetic field model (c.f. Paper I where the misalignment process was applied to non-force-free-field and MHD models).

A method to determine the control points $z_{i}$ 's is now discussed in terms of minimizing the difference in direction between the 3D coronal-loop tangents $\left[\mathbf{B}_{\text {obs }}(\mathbf{R})\right]$ and the associated direction of a theoretical magnetic-field model $\left[\mathbf{B}_{\text {theo }}(\mathbf{R})\right]$. $\mathbf{B}_{\text {obs }}$ is the tangent vector of the curve defined by Equation 3. This follows the concept developed in Paper I. The change in this paper again is that all four of the control points have a variable z-component. At a 3D position $(\mathbf{R})$ in the corona, we define the misalignment angle at a point, $\mu(\mathbf{R}), 0 \leq \mu \leq \pi$, (DeRosa et al., 2009; Aschwanden and Malanushenko, 2013) as

$$
\mu(\mathbf{R})=\cos ^{-1}\left[\frac{\mathbf{B}_{\text {theo }}(\mathbf{R}) \cdot \mathbf{B}_{\text {obs }}(\mathbf{R})}{\left|\mathbf{B}_{\text {theo }}(\mathbf{R})\right|\left|\mathbf{B}_{\text {obs }}(\mathbf{R})\right|}\right],
$$

where the angle $\mu$ is defined in terms of the spatial position, $\mathbf{R}$, and the directions of the 3D curve representing the loop and the model field strength, evaluated at that point. For the entire loop, a characteristic misalignment angle is define by the equation

$$
\xi=\frac{1}{\Gamma} \sum_{k=1}^{\Gamma} \mu\left(\mathbf{R}_{k}\right),
$$

where the sum is over $\Gamma=100$ equi-spaced points along a field line and $\Gamma$ has the same value for all loop lengths. Hence for the $j^{\text {th }}$-loop, the characteristic misalignment angle $\xi_{j}=\xi_{j}\left[z_{j 1}, z_{j 2}, z_{j 3}, z_{j 4}\right]$ is a function of the $z_{j}$ 's. The best $3 \mathrm{D}$ Bézier coronal fit, established by the minimum of $\xi_{j}$, determines the $z$-coordinate position of the loop, that is defined by

$$
z\left[z_{j 1}, z_{j 2}, z_{j 3}, z_{j 4}\right]=(1-u)^{3} z_{j 1}+3 u(1-u)^{2} z_{j 2}+3 u^{2}(1-u) z_{j 3}+u^{3} z_{j 4} .
$$

Hence, given a 3D magnetic-field extrapolation model $\left[\mathbf{B}_{\text {theo }}^{m}(x)\right]$, where $m$ is the model number, and using the 3D Bézier loop construction, we can construct the 
characteristic misalignment angle for the $j^{\text {th }}$ loop and $m^{\text {th }}$ theoretical magneticfield model assuming values for $z_{i}$ 's :

$$
\xi_{j}^{m}\left[z_{j 1}, z_{j 2}, z_{j 3}, z_{j 4}\right]=\frac{1}{\Gamma} \sum_{k=1}^{\Gamma} \cos ^{-1}\left[\frac{\mathbf{B}_{\text {theo }}^{m}\left(\mathbf{R}_{k}\right) \cdot \mathbf{B}_{\text {obs }}\left(\mathbf{R}_{k}\right)}{\left(\left|\mathbf{B}_{\text {theo }}^{m}\left(\mathbf{R}_{k}\right)\right|\left|\mathbf{B}_{\text {obs }}\left(\mathbf{R}_{k}\right)\right|\right)},\right.
$$

where $\mathbf{R}_{k}=\mathbf{R}_{k}\left[z_{j 1}, z_{j 2}, z_{j 3}, z_{j 4}\right]$.

For a particular model $(m)$, the final set of values for $\left[z_{j 1}, z_{j 2}, z_{j 3}, z_{j 4}\right]$ are determined by the values that minimize the set $\xi_{j}^{m}$, i.e.

$$
\xi_{j}^{m}\left[z_{j 1}^{*}, z_{j 2}^{*}, z_{j 3}^{*}, z_{j 4}^{*}\right]=\min _{z_{1}, z_{2}, z_{3}, z_{4}}\left[\xi_{j}^{m}\left[z_{j 1}, z_{j 2}, z_{j 3}, z_{j 4}\right]\right] .
$$

(Figure 8 of Paper I gives a visual illustration of this single-loop minimization process.) The best fit for the four parameters (given by $z_{j 1}^{*}, z_{j 2}^{*}, z_{j 3}^{*}, z_{j 4}^{*}$ ), for the $j$ th loop for magnetic field model $m$, is determined using the Powell method which is a conjugate-direction method to find the minimum in multidimensional spaces (in our case, it's a four-dimensional space) (Presset al., 1988). The convergence criteria is set by specifying the fractional tolerance of $\xi_{j}^{m}$. The failure to decrease by more than the fractional tolerance in one iteration signals completeness of the search algorithm. (We implement the process with the IDL routine POWELL with $F T O L=1 \times 10^{-4}$ ). The asterisk values for the $z_{i j}$ s are given to emphases that these z-values are the resultant values for the Powell minimization for a particular loop $(j)$ and a particular magnetic model $(m)$. Using the best fit parameters $z_{j i}^{*}$ 's, we define a 3D global misalignment-angle parameter $(\Phi)$ using all of the $N$ loops for each model:

$$
\Phi[m]=\frac{1}{N} \sum_{j=1}^{N} \xi_{j}^{m}\left[z_{j 1}^{*}, z_{j 2}^{*}, z_{j 3}^{*}, z_{j 4}^{*}\right] .
$$

We define this as a 3D measure of the goodness-of-fit for each model $m$. The optimum magnetic model representation for the corona is given by the model that has the smallest misalignment angle, i.e. satisfies $\min _{m}[\Phi[m]]$. Assuming that all the models extrapolate the same photospheric vector magnetic field then this procedure allows a selection of the best model that agrees with the observed EUV coronal loops.

Since $\Phi[m]$ is magnetic-field model dependent and if we employ a potential magnetic field model, the resulting value of $\Phi[$ potential $]$ will reflect a measure of active-region nonpotentiality (Falconer, Moore, and Gary, 2008). In Section 5 , we will explore this relation by comparing the $\Phi[$ potential $]$ with known nonpotentiality measures. The variation of $\Phi[$ potential] with active regions may be directly indicative of the heating process variation if the EUV loops are associated with foot-point heating through the twisting and braiding of the loop sub-elements.(e.g. see Aschwanden (2004), §9.3). Since the specific values of $\Phi[$ potential $]$ might depend on the total current in a highly non-linear manner, a future study of the relation of $\Phi[$ potential $]$ to the X-ray emission, from an active region, would be a prudent step in determining whether the relation $\Phi[$ potential $]$ and heating of the corona are correlated. 


\section{Potential Magnetic Field Comparison Case}

In this section, we use a known potential magnetic field as a comparison case to show the fitness of the Occult- 2 code of loop extraction with a known input, and to show that a set of $2 \mathrm{D}$ projected loops can be used to restructure the loops back into 3D space. We first compare Occult-2 tracings to the actual projected (2D) field lines from which the rasterized image was generated. The method uses the magnetic-field lines which are calculated using dipole fields and a resulting image of the magnetic-field lines are produced. The magnetic field is a multi-dipole representation of active region AR 11117 using 17 dipoles to obtain an approximation of the vertical magnetic field using a 10-pixel smooth HMI magnetogram (with a FOV of $\sim 350$ by $250 \operatorname{arcsec}^{2}$ and a spatial resolution of 0.5 arcsec). A set of 201 field lines were calculated in a rectangular cuboid with a height of 250 arcsec. The starting foot points for the field lines are scattered evenly over the distribution of absolute field strengths, above 250 Gauss. The resulting field lines, viewed vertically downward, of the active region was rasterized into an image of 700 by 500 pixels. The resulting image was smoothed with 2D Gaussian smoothing function of a half-width of 10 pixels and random noise between 0 and $2 \%$ was added to create the synthesized $171 \AA$-like filtergram (Figure 2, left panel).

The Occult-2 loop recognition software (version dated 2013/06/20) was applied to the synthesized filtergram. The parameters applied in the Occult-2 program had a minimum 30-pixel radius of curvature allowable and a minimum loop length of 25 pixels with the bandpass filter constants of $n s m 1=10$ and $n s m 2=12$. Occult- 2 retrieved 100 loop segments that was above the program's threshold condition (right panel, Figure 2). Each of the traced loop segments were fitted with a 2D Bézier spline such that the 1st and 4th control points were at the end points of the Occult-2 lines. The curve fitting program minimized the RMS distance between the curve and the Bézier spline employing 10-equally spaced points along both curves. The $n=10$ Euclidean distances along the $2 \mathrm{D}$ Bézier curve (f) and the 2D Occult-2 loop (r) are used to minimize the following expression:

$$
\chi=\left[\frac{1}{n} \sum_{\nu=1}^{n}\left[\mathbf{f}\left(u_{\nu}\right)-\mathbf{r}_{\nu}\right]^{2}\right]^{1 / 2} .
$$

Since the Bézier curve is nonlinear with respect to its parameter, the values $u_{\nu}$ have to be derived such that they give equal spacing along the arclength of the spline.

Figure 3 shows the resulting Bézier spline, of the fitted Occult-2 loops, overlaying the set of field lines used to generate the synthesized filtergrams. The few small mismatches between the spline fit and the field line results from overlapping loops and image noise. Overall the 2D Bézier splines from the Occult-2 tracing fits well the potential loops. Hence Occult-2 performs well on synthesized image of Figure 2 where the loop locations are known.

The 2D Bézier splines were extended to 3D using the minimum misalignment angle to determine the vertical heights of the four control points as described in section 2. A computer algorithm was implemented to determine the heights of 

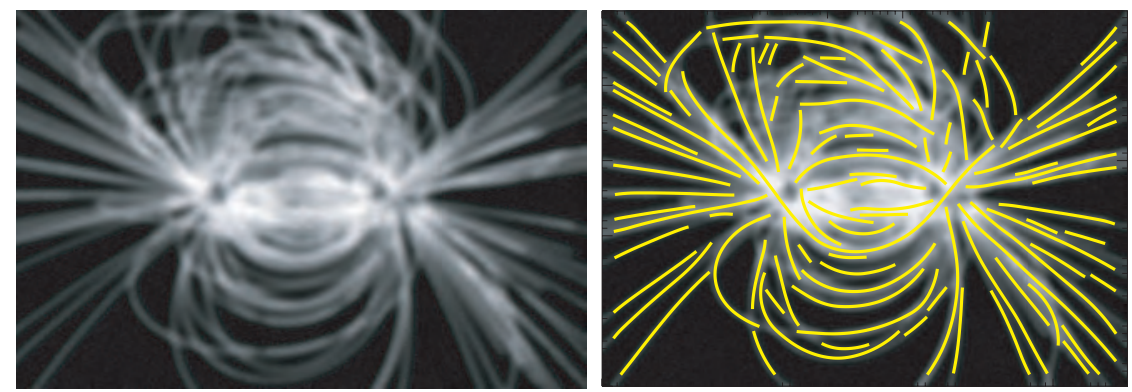

Figure 2. A synthesized filtergram (left panel) was generated using 201 potential field lines which were rasterized, Gaussian blurred, and noise added to the image. The 100 curves (yellow) obtained by the automated loop tracing routine, Occult-2, are shown overlaid on the synthesized filtergram (right panel)

control points, $\mathbf{P}_{1} \mathbf{P}_{2} \mathbf{P}_{3}$ and $\mathbf{P}_{4}$, that minimizes the angular differences between the cubic Bézier curve tangents and the potential magnetic field direction at 10 points per line. For each 3D curve the average misalignment angle is used to color code the loops plotted in Figure 3 and 4 . Figure 4 shows the resulting height structures derived in the cuboid as viewed from south and the west. The results show that there is no correlation of the misalignment angle with height. The distribution of the number of loops with misalignment angle shows that $33 \%$ of loops have a misalignment less than 1.8 degrees, while $50 \%<2.3$ degrees (median), and $80 \%$ less than 3.5 degrees. For this test, the average misalignment angle for all the loops is $\Phi[$ test $]=3.6$ degree. This value is different from zero mainly as a result of small errors in the loop identification. For the potential results shown in Figure 2, there are many relatively straight and many short segments which are more problematic for the minimization code since, as seen in Figure 4, these are restrained less in the geometric fitting. A very short line can be tangent to many more of the potential field lines of various heights. These probably also have contributed to the non-zero value of $\Phi[t e s t]$, but we have not tested their contributions. Figure 4 shows the derived loop structure agrees with the potential field lines in most cases. This test case shows the capability of the process and provides the limiting value of $\Phi$ that can be expected for the parameters used in the present study.

\section{Linear Force-Free Field Results}

Applying the misalignment angle method, this section describes results using the magnetic-field models which employ three multiple LFFF dipole models (Equation 4) for the active region AR 11117. We give the resulting field line comparisons with AIA $171 \AA$ A coronal filtergrams and the statistical comparisons of misalignment angles for 3 linear, constant- $\alpha$ force-free-magnetic-field cases. These cases are for a diverse set of $\alpha$ 's with $\alpha=\left\{-\alpha_{\max }, 0,+\alpha_{\max }\right\}$ where $\alpha_{\max }=1 /[$ FOV width] $($ Gary, 1989).

Using the active region AR 11117 , a smoothed- $B_{\text {los }}$ magnetogram $\left[B_{\text {smooth }}\right]$ is derived via Gaussian smoothing $\sigma=10$ pixels, where $\sigma$ is the equivalent height 


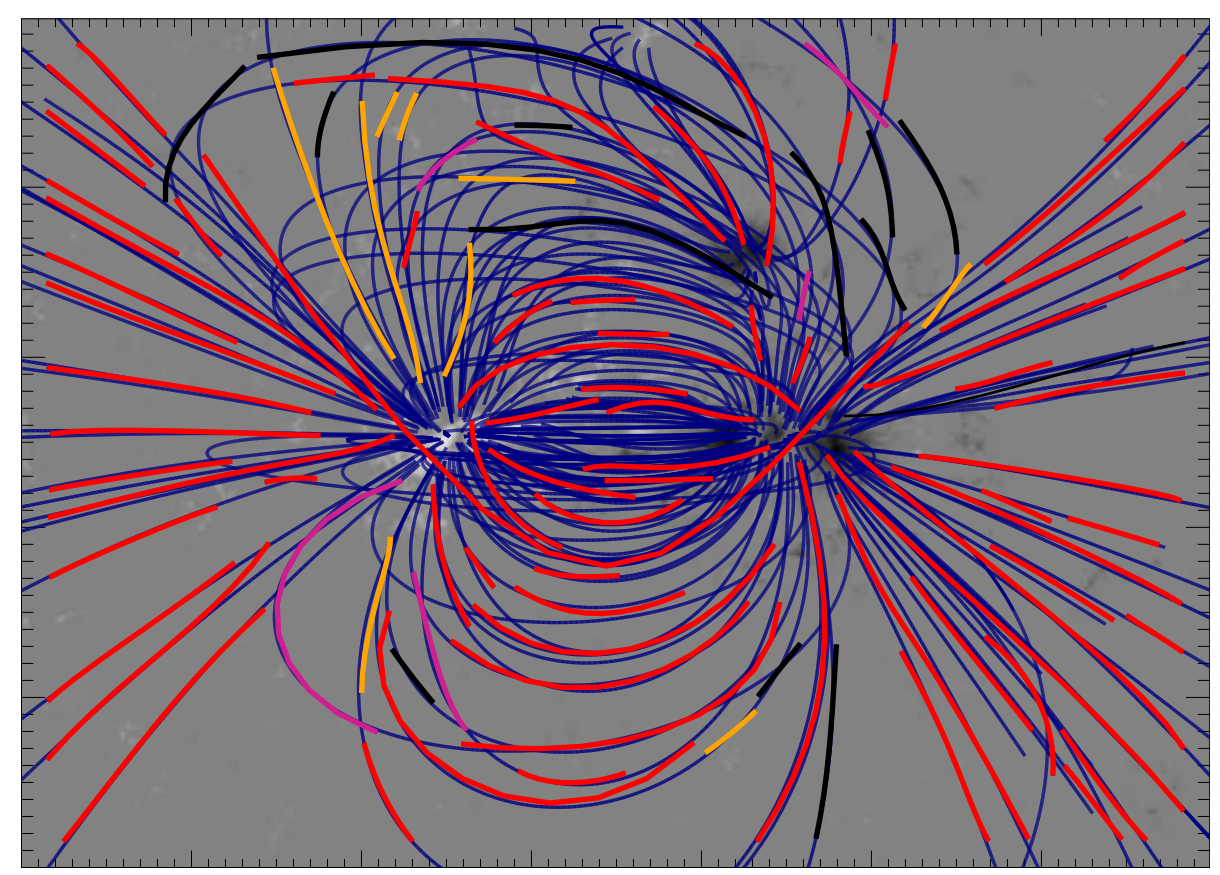

Figure 3. The 2D Bézier curves that were fitted to the Occult-2 curves are shown overlaying the original potential field lines (blue) which generated the rasterized image. Color code for the Bézier curves give the fitness of the 3D Bézier curves $i$ as defined by the total misalignment angle $\xi[i]$,in degrees, over the loop (red, $0 \leq \xi[i] \leq 15$; orange, $15<\xi[i] \leq 30$; purple, $30 \leq \xi[i] \leq 60)$. The small set of lines (black) were not used, because they where initially identified, without reference to the calculated magnetic field lines, as false-positive curves, although in retrospect seem appropriate for $3 \mathrm{D}$ analysis.

in the corona which would have similar field values. Picking the strongest magnetic extrema (peak values $\geq 250$ Gauss) of the absolute value of the smoothed magnetgram, the positions $\left(x_{i}, y_{i}\right)$ of the 17 locations are used as the locations of the LFFF constant- $\alpha$ dipoles. The number of dipoles is dependent on the number of major magnetic islands (Figure 5). We set all of the dipoles at $15 \mathrm{Mm}$ below the photosphere to obtain the dipole fields with the approximate width of the magnetic islands at the photosphere and then calculated the dipole strengths $M_{i}$, such that the vertical field values of all the dipoles $B_{z}\left(x_{i}, y_{i}, z=0\right)$ is equal to the smooth magnetogram value at $\left[x_{i}, y_{i}\right]$ (Figure 5 ). This is accomplished by solving for the minimum RMS difference in $B_{\text {smooth }}$ and $B_{z}$ at $\mathrm{z}=0$. This procedure gives the magnetic-field model that is used in this section.

A set of 2D Bézier splines is fitted to the Occult-2 traced loop results (shown in Figure 6) and we extend them to 3D Bézier splines and derive the heights of their control points by using three linear force-free magnetic-field models, with the method described in the last section. The $\alpha$ values used $\left(-\alpha_{\max }\right.$, 0 (potential), and $+\alpha_{\max }$ ) provide three different global twisting of the field lines. The LFFF multi-dipole magnetic field approximation uses the dipoles defined by Equation 4. The results for the derived heights of the traced loops are shown in Figure 7 along with the appropriate set of constant- $\alpha$ field lines. 

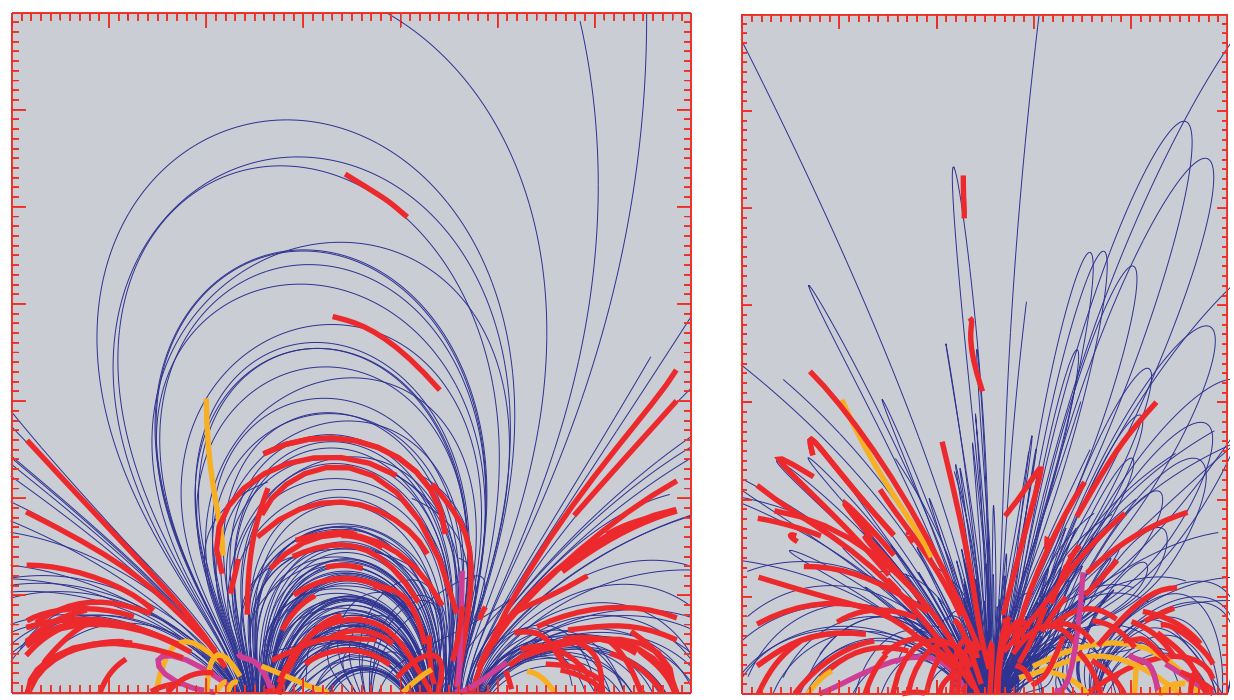

Figure 4. Using extended 3D Bézier curve derived from the synthesize filtergram and the originating potential-field model, the z-components of the control points were determined. The two panels (front and side views) show the derived height result using the minimization of the misalignment angle. The authentic potential field lines (blue) are shown and the color coding is the same as Figure 3.
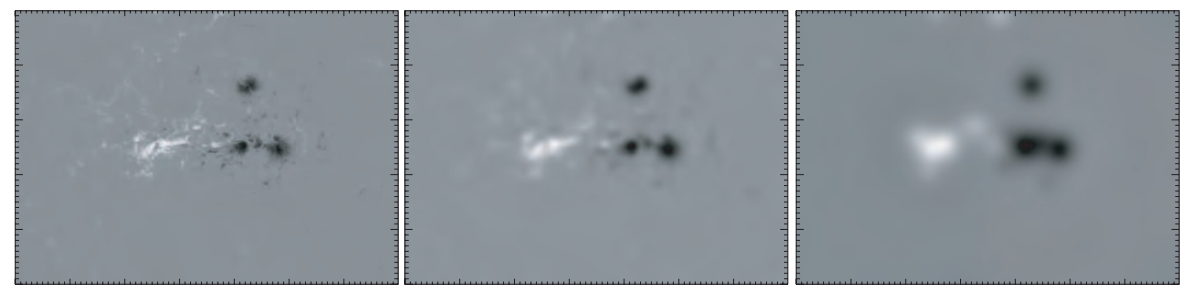

Figure 5. The HMI magnetogram for active region AR 11117 on 25 October 2010 is shown (left panel) with a FOV of 350 by $250 \operatorname{arcsec}^{2}$ (or 700 by 500 pixels). A 10-pixel Gaussian smoothing function is applied to the HMI magnetogram (middle panel). The largest maxima and minima of the smoothed magnetogram is used to generate the magnetic-field model from a set of 17 dipoles. This modeled field magnetogram is show in the right panel.

Some of the derived loop trajectories appear open-like although all field lines of our simple magnetic model are closed The total misalignment angle for the three cases are $\Phi\left(-\alpha_{\max }\right)=10.8, \Phi(0)=8.3$, and $\Phi\left(+\alpha_{\max }\right)=11.9$ degrees. These values are consistent with the visual comparison of the field lines for each model to the filtergram. For example, the large loop system in the south of AR 11117 tends to agree more with the $\alpha_{\max }=0$ solution that has the smallest $\Phi$-value. Even though the field lines show differences for the three cases, the misalignment-angle results may not be significant. The active region cannot be modeled adequately with a LFFF model to reduce the misalignment angle below 5 degrees; furthermore, $\alpha$ must be a function of position in the solar corona and is not constant as assumed. However these $\alpha$-results show some similarities that 

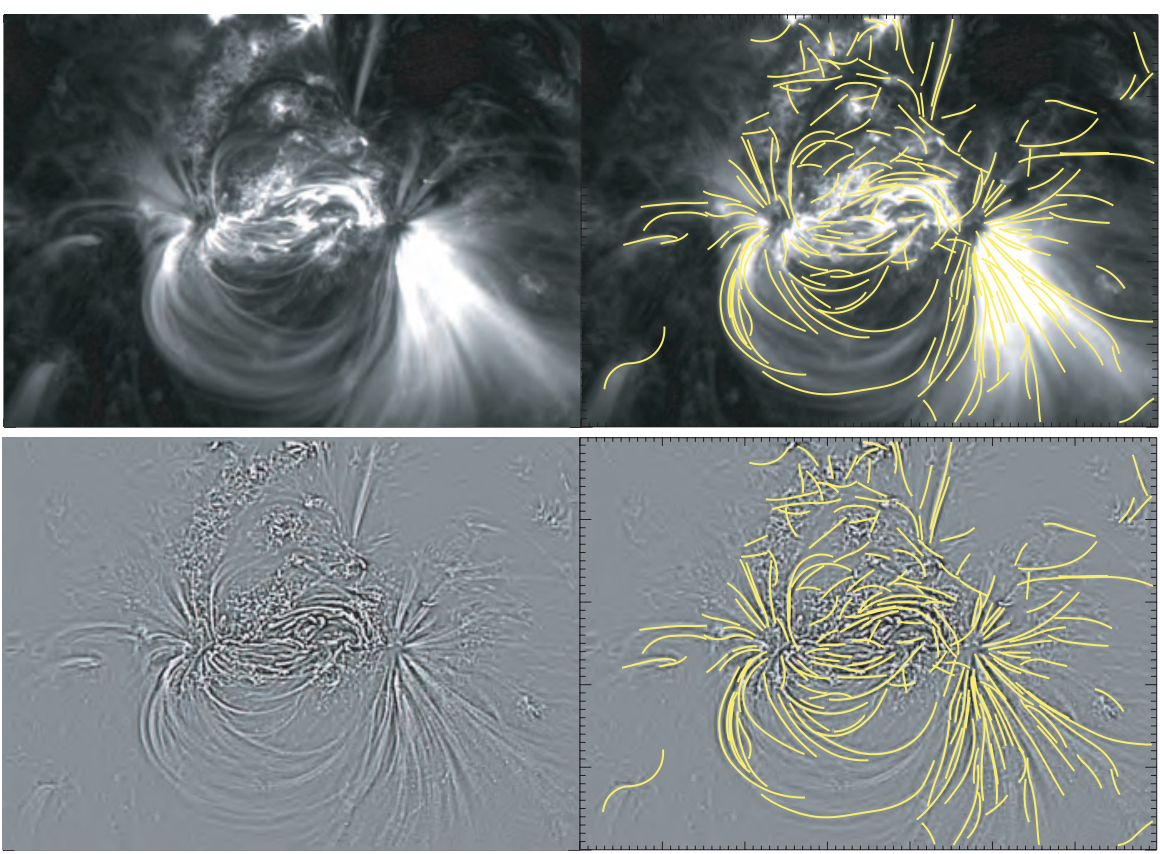

Figure 6. The AIA $171 \AA$ filtergram for active region AR 11117 on 25 October 2010 is shown (left panel) with a FOV of 350 by $250 \operatorname{arcsec}^{2}$. The lower left panel is a high frequency bandpass highlighting the loop structures. The Occult-2 identified loops are shown on the right over the corresponding image to the left. There is a set of false-positive identifications, for example, near the top of the figure, which are manually excluded in the analysis of this active region.

probably infer some information of the actual 3D physical coronal structure. Figure 4 shows a few open field lines for each case. The statistical distribution of the 3D Bézier loops derived from the minimization of the misalignment angles using a potential magnetic-field model show that the misalignment angles are independent of height, most derived loops are located near the photosphere, most loops have misalignment angles $<9$ degrees, and most loops are not closed loops. The distribution of the number of loops with misalignment angles shows that $33 \%$ of the loops have a misalignment less than $5.0,3.5$, or 6.5 degrees for the three $\alpha$ 's, $\left(-\alpha_{\max }, 0,+\alpha_{\max }\right)$, respectively $)$, while $50 \%<(8.4,5.1,8.9)$ degrees, and $80 \%$ less than $(16.0,12.7,19.1)$ degrees.

\section{Three Observed Active Regions with AIA and HMI data}

This section compares the results for the derived field lines using a potential model for active regions: AR 11117, AR 11158, and AR 11283 (Table 1). The resulting loops and field-line plots in 3D are presented in top, front, and side views. The statistical comparison is given for the results of the misalignment angles in terms of the misalignment angle dependency on height, loop height locations, and an estimation of the number of open to closed loops (where 

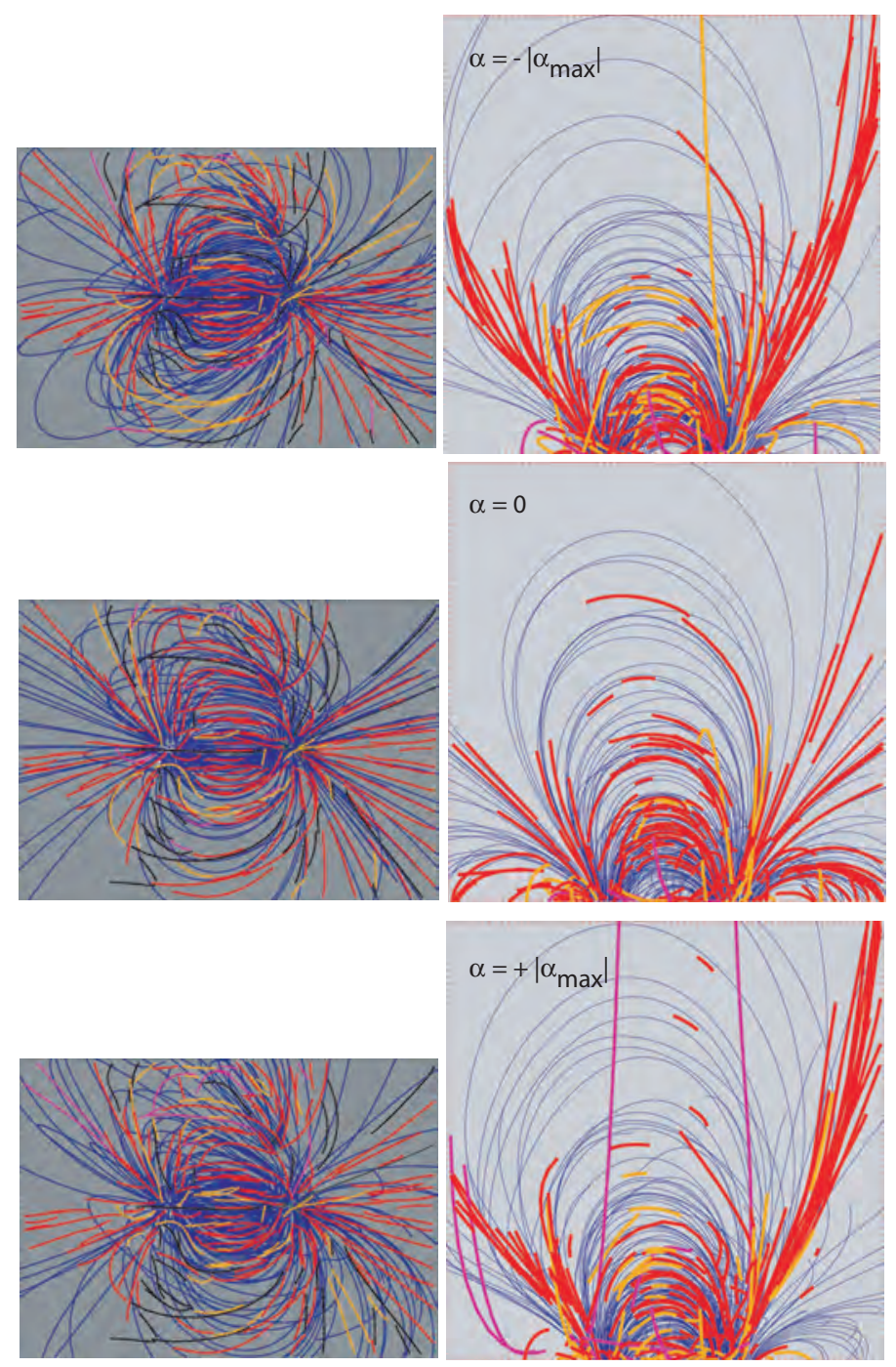

Figure 7. The resolved heights of the coronal structure for AR 11117 for three LFFF models are shown with a FOV of 350 by $250 \operatorname{arcsec}^{2}$. The left panels show the Occult-2 resolve loops overlaid on the linear force-free field with $\alpha$ values of $-\alpha_{\max }, 0$, and $+\alpha_{\max }$, from top to bottom. The right panels show the 3D resolved loops as viewed from the south. The 3D Bézier curves $(i)$ are color coded as a function of their total misalignment angle $\xi[i]$ (in degrees) over the loop (red, $0 \leq \xi[i] \leq 15$; orange, $15<\xi[i] \leq 30$; purple, $30 \leq \xi[i] \leq 60$; and black, $60<\xi[i])$. 


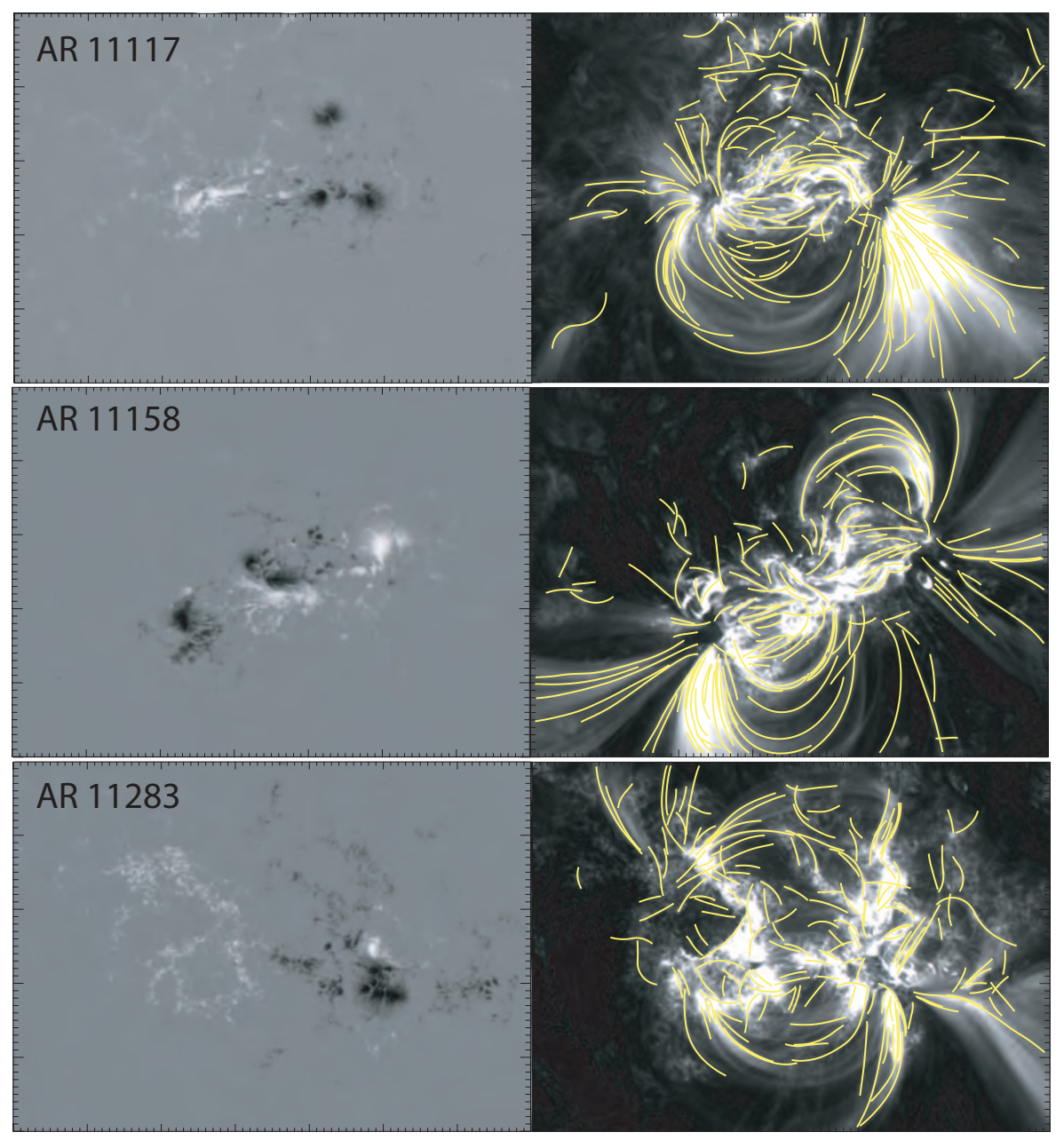

Figure 8. The HMI magnetogram and the AIA $171 \AA$ filtergram with the Occult-2 traced loops for three active regions: AR 11117, AR 11158, AR 11283 (see Table 1).

open-like loops are determined by $\left|z_{1}-z_{4}\right|>100 \operatorname{arcsec}$, where $z_{1}$ and $z_{4}$ are defined by Equation 3). The distribution of the misalignment angles per loop is present for each active region in Table 2. The visual comparison of the $3 \mathrm{D}$ fitted coronal loops to the distribution of limb loops is shown, with the limb observation occurring 5 or 6 days later than the on-disk observations (see Table $1)$.

Figure 8 shows the HMI magnetograms for the three regions with a FOV of 350 by $250 \operatorname{arcsec}^{2}$. The AIA $171 \AA$ filtergrams are shown with the Occult-2 traced coronal loops. Each traced loop is fitted with a 2D Bézier curve and the minimization of the misalignment angle process leads to the 3D locations of the loops as shown in Figure 9. The statistical distribution of the 3D Bézier loops derived from the minimization of the misalignment angles using a potential magnetic- 
field model gives the following results: i) the variation of misalignment-angle values are independent of height, ii) most of the loops structure are located near the photosphere, iii) the average loops have misalignment angles $<12$ degrees, and, iv) most loop segments are not associated with open loops. The percent of the total number of loops for the three active regions that have open-like loops are $18 \%, 23 \%$, and $30 \%$ for AR 11117, AR 11158, AR 11283, respectively. The misalignment angle data for the three regions is given in Table 2 . The distribution of the number of loops as a function of the misalignment angle shows that $33 \%$ of loops have a misalignment less than 5.5, 7.7, or 5.5 degrees for the three active regions AR 11117, AR 11158, and AR 11283, respectively, while $50 \%<(10.2$, $11.5,8.1)$ degrees, and $80 \%$ less than $(21.0,28.0,24.4)$ degrees. For this test, the overall misalignment angle for all the loops are $\Phi[A R 11117]=13.0$ degrees, $\Phi[11158]=15.9$ degrees, $\Phi[$ AR11283] $=13.7$ degrees.

The three active regions studied appear on the limb 5 or 6 days after the near-central-meridian observation from which we have derived the loop heights. The height of the EUV loops above the photosphere can be directly observed on the limb. In Figure 10, we compare the on-disk derived heights and the limb observed heights of the coronal loops. Since the active region evolves during the several days of transit time to the limb, the comparison is presented as only a homologous study. The distribution and location of the derived coronal loops are similar even though the magnetic-field model is potential. Overall the comparison gives added credulity to this method of deriving the coronal loops heights and will acquire improvement with the employment of non-LFFF (NLFFF) or MDH magnetic-field models, from which the critical magnetic free energy can be derived.

The total misalignment angle derived using a potential magnetic-field model might be a gauge of the total free energy. To investigate this possibility we considered the total-misalignment-angle correlations with i) the largest x-ray flares occurring within 24 hours of the on-disk observations and ii) the free-energycontent measure ${ }^{L} W L_{S G}$ (Falconer, Moore, and Gary, 2002; Falconer, Moore, and Gary, 2008; Falconer, Moore, Gary, and Adams, 2009). The results are shown in Table 2. Assuming that the x-ray flare type is indicative of the three active regions' degree of nonpotentialilty, there appears no obvious connection within this limited data set. However Falconer's free-energy-content measure shows a correlation with the misalignment angle. Hence, more study is needed to verify if the total misalignment angle is a direct measure of the nonpotentiality of active region and is useful for space weather predictions. If the major nonpotentiality of the magnetic field is localized, the misalignment angle may only weakly reflect the nonpotentiality since the longer, higher coronal loops are seen in the $171 \AA$ filtergrams.

\section{Summary and Conclusion}

The Occult-2 loop-tracing program can give some false-positive detections, however, since the number is small, these tracings can be visually found and manually

eliminated (e.g. Figure 3). The process of minimizing the misalignment angle is 

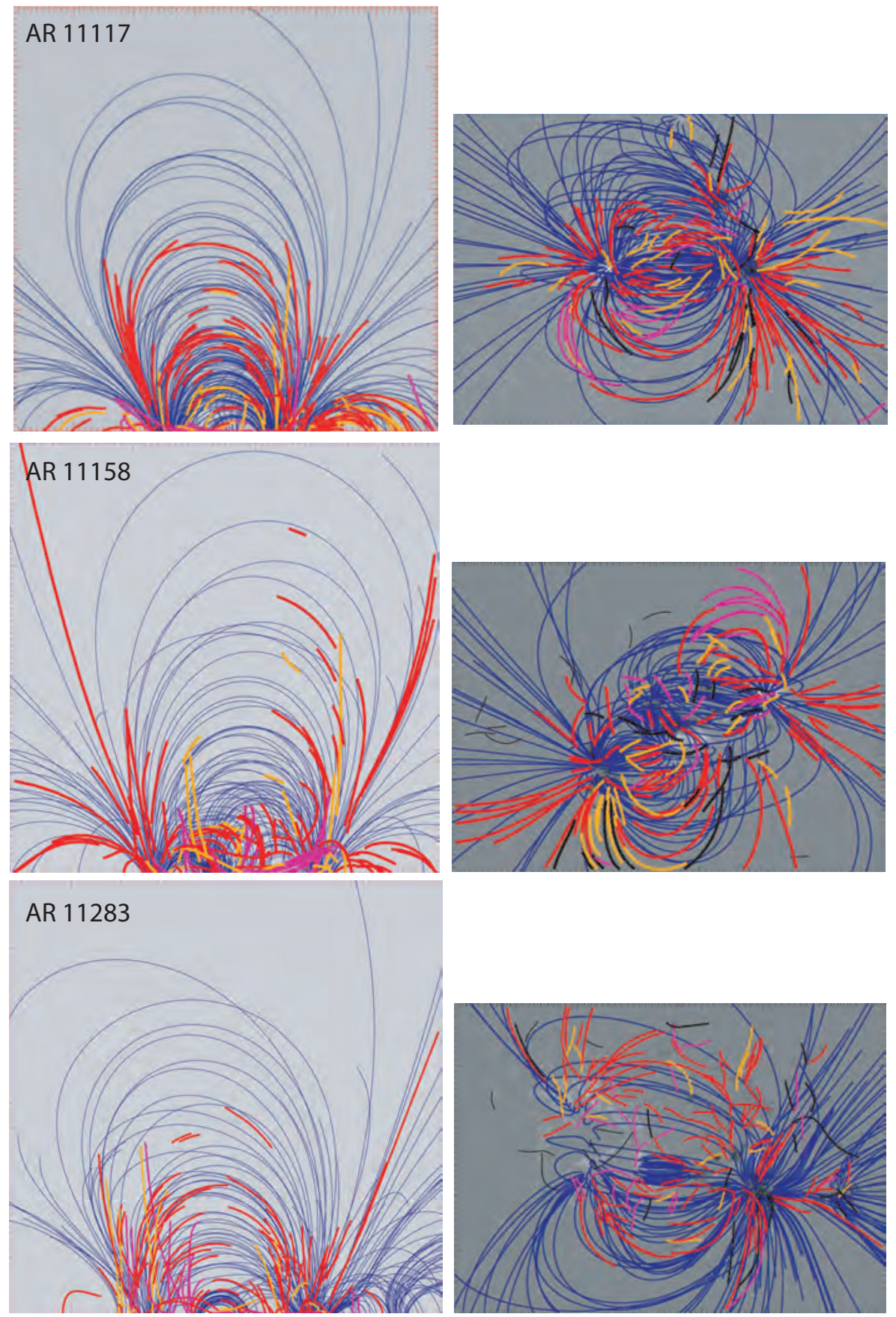

Figure 9. Top and side views for active regions AR 11117, AR 11158, and AR 11283, from top to bottom. The misalignment-angle color coding is the same as Figure 7. The heights of the loops are similar for the three active regions which are conferred in limb views (Figure 10). 
Table 2. Comparison of the three active regions - AR 11117, AR 11158, and AR 11283. The total misalignment angle, $\Phi[$ potential $]$, is given in degrees. The percent of the number of loops which have their individual average misalignment angle less than a particular angle is given for three values. The number of dipoles used in constructing the potential magnetic-field model using the minimization of misalignment angles is given. The x-ray type of the largest flare within 24 hours of the on-disk observation is given in the next to last line with the time of the flare (UT). The last line has the free-energy-content measure ${ }^{L} W L_{S G}$ obtained from a line-of-sight magnetogram and defined by ${ }^{L} W L_{S G}=\int\left|\nabla B_{\text {los }}\right| d l$, where $\nabla B_{\text {los }}$ is the transverse gradient of the line-of-sight magnetic field and the integral is the line integral over all neutral-line intervals on which the potential transverse field computed from the magnetogram is $\geq 150 \mathrm{G}$. We note the active region AR 11158 produced an X2-class flare (the Valentine's Day Flare) which was produced just two hours after our selected time window (Tarr, Longcope and Millhouse, 2013).

\begin{tabular}{cccc}
\hline Active Region & AR 11117 & AR 11158 & AR 11283 \\
\hline$\Phi[$ potential $]$ & 13.1 & 15.9 & 13.7 \\
$\xi<6$ degrees & $37 \%$ & $29 \%$ & $35 \%$ \\
$\xi<12$ degrees & $58 \%$ & $53 \%$ & $67 \%$ \\
$\xi<24$ degrees & $86 \%$ & $78 \%$ & $80 \%$ \\
No. Dipoles & 17 & 31 & 52 \\
Largest flare & $\mathrm{C} 2(22: 06)$ & $\mathrm{M} 6(17: 28)$ & $\mathrm{X} 2(22: 12)$ \\
${ }^{L} W L_{S G} \mathrm{G}$ & $19 \times 10^{3}$ & $39 \times 10^{3}$ & $17 \times 10^{3}$ \\
\hline
\end{tabular}

heavily dependent on the magnetic-field model, however, this dependency is a positive feature in that it gives a measure of the strength of the magnetic-field model to fit the EUV observed loops. In the regions studied we have not had any traced-coronal-loops structures that needed higher order Bézier splines, hence we believe that the cubic splines can represent efficiently almost all observed EUV coronal loops. The conclusion of this study is that the $3 \mathrm{D}$ cubic splines method described in this paper can provide i) insight of the coronal structure independent of heliostereoscopy, ii) provide a powerful method of comparing competing magnetic-field models, and iii) constrain the magnetic structure of the corona. Our 3D identification process and the application of employing Bézier curves described can be used to improve and refine MHD modeling efforts by identifying subregions where the model and observations diverge, and, hence, infer the direction for model improvement. In particular, this new method can provide a process to improve the global structure of the complete magneticfield structure for active regions. The process of cross-checking the models with coronal-loop structures simulates a type of upper-boundary condition for which models must concur.

As stated in Paper I, similar investigations have been performed by other research groups. For example, Malanushenko, Longcope, and McKenzie (2009) and Malanushenko, Yusuf, and Longcope (2011) use a piece-wise cubic spline 

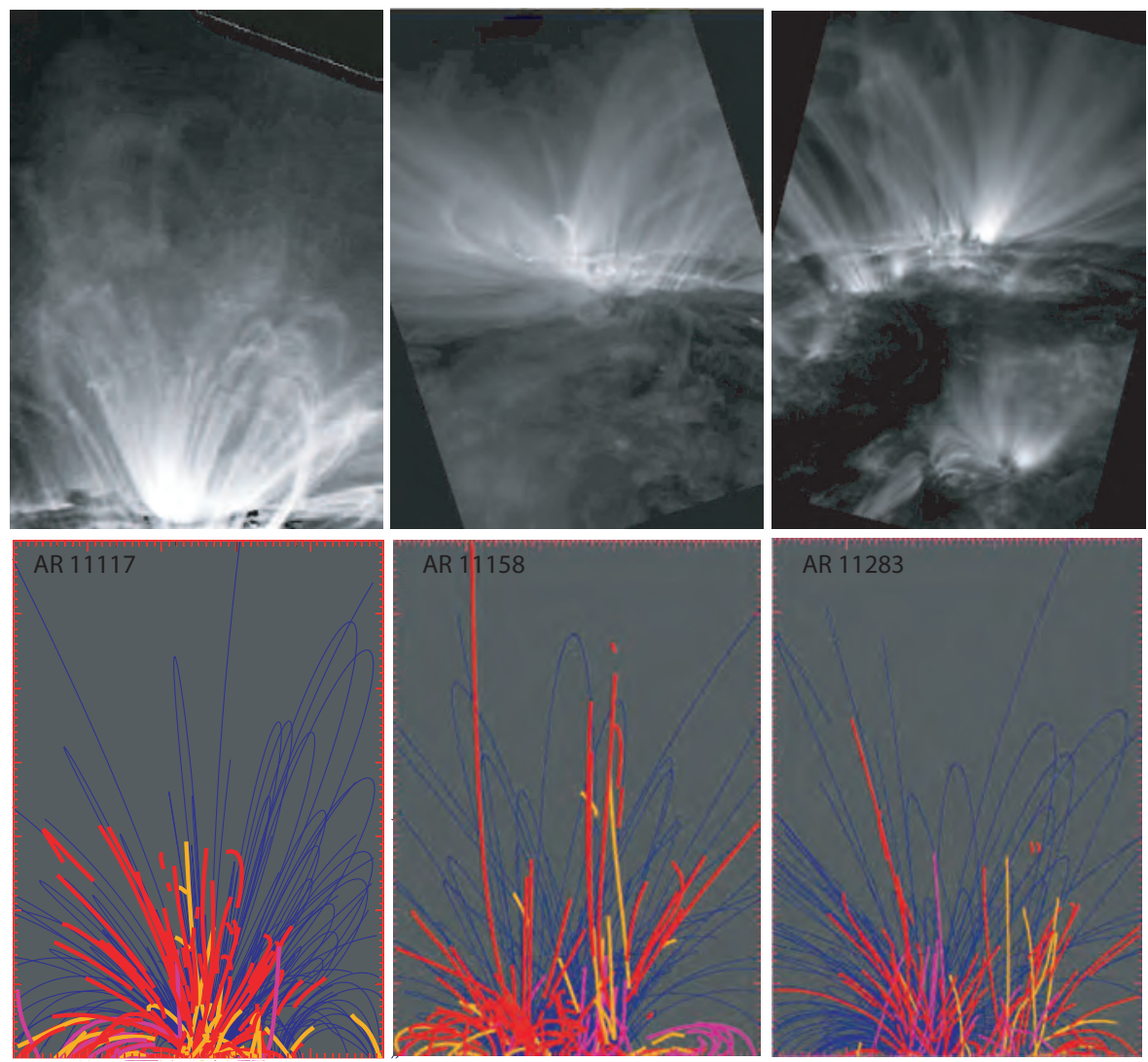

Figure 10. Side views for the loops for active regions AR 11117, AR 11158, and AR 11283 are shown and compared with the AIA $171 \AA$ loops as seen on the limb. The misalignment-angle color coding is the same as Figure 7. The heights for the reconstructed loops are similar to the observed heights when the active region passes the limb several days later (see Table 1 ). The AIA observations and the magnetic field lines side views are scaled the same (250 by 350 $\operatorname{arcsec}^{2}$.)

function for a coronal loop and searched for linear force-free field solutions curves in both its force-free parameter and height for each best-fit field line to infer the twist of the lines. Various calculations have been performed to select field lines from different LFFF and NLFFF models to determine the free parameter $(\alpha)$ which gives the best fit of the coronal lines (Lim et al., 2007; Lopez Fuentes, Klimchuk and Demoulin, 2008; Malanushenko, Longcope, and McKenzie, 2009; Aschwanden and Malanushenko, 2013; Aschwanden et al., 2012b). STEREO investigations have provided unique 3D reconstructions to allow testing of various extrapolation models (Aschwanden and Malanushenko, 2013; Aschwanden et al., 2012b). Our approach presented in Paper I and here is similar to the STEREO method investigated by Aschwanden (2013a); however we have used a cubic Bézier curve fitting approach with applications for distinguishing competing magnetic-field models. 
The importance of employing coronal-loop images in selecting the magneticfield model arises in part from the difficulty of measuring the photospheric magnetic field for magnetic field extrapolation. Wiegelmann and Inhester (2010) and Wiegelmann et al. (2010) addressed the implication of the vector-magnetogram errors for deriving a nonlinear force-free magnetic-field model. Our method also improves the knowledge for the coronal magnetic-field modeling and overcomes the defects of not being able to sufficiently measure the field from radio or optical observation, where the height of the observations on the disk is problematic to determine. The uncertainties in the magnetic field can be ameliorated by using the coronal-loop information on the 3D structure of the magnetic-field lines and connectivity, hence there is a necessity to map the coronal loops and extract their information for an improved assessment of magnetic-field models (e.g. see DeRosa et al. (2009)).

In summary, we have presented a method to derive the height of observed EUV coronal loops based on the extension of the cubic Bézier splines to represent the EUV coronal loops of an active region and by using a magnetic-field model. The technique uses only four points per field line, and employs the computer-efficient and rapid tracing algorithm (Occult-2). Since the coronal loops are used as surrogates of magnetic-field lines, the Bézier mapping can restrict the magneticfield models derived from extrapolations of magnetograms to those admissible and inadmissible, since the magnetic-field extrapolations must satisfy not only the lower boundary conditions of the vector field, the vector magnetogram, but also must have a set of field lines that satisfies the additional conditions in the volume, akin to supplying an upper boundary condition. The tool and program are important in determining the magnetic-field models for the solar atmosphere which are crucial in determining the overall dynamics of the solar atmosphere. We have applied our technique to three active regions using a potential magnetic-field extrapolation model to demonstrate its capabilities. For active region analysis, the generalizations of this technique of coronal-loop identification and misalignment-angle analysis can also be used for improving the 3D modeling of the solar coronal magnetic field (e.g., an MHD calculation), by incorporating minimization of the misalignment angle into the iterations. This process of including coronal imagery to improve the understanding of the magnetic field will eventually improve the understanding of the solar atmosphere dynamics.

Acknowledgements The research in part was funded by the NSF's Division of Atmospheric and Geospace Sciences under the SHINE (Solar, Heliospheric, and Interplanetary Environment) program for SHINE: Analysis of Solar Active Region Energetics Based on Non-Force Free Coronal Magnetic Field Extrapolation (AGS 1062050) (http://www.shinecon.org/). We wish to thank Dr. D. A. Falconer (CSPAR,UAHuntsville) for making available the free-energycontent measures.

\section{References}

Aschwanden, M.J.: 2004, Physics of the Solar Corona An Introduction, Springer, NY. 
Aschwanden, M.J.: 2010, Solar Phys. 262, 399, ADS:2010SoPh..262..399A, doi:10.1007/s11207-010-9531-6.

Aschwanden, M.J.: 2013a, Astrophys. J. 763, 115.

Aschwanden, M.J.: 2013b, Solar Phys. 287, 369, doi:10.1007/s11207-012-0203-6.

Aschwanden, M.J., De Pontieu, B, Katrukha, E. A.: 2013, Entropy, open access, doi:10.3390/e15083007.

Aschwanden, M.J., Malanushenko, A.: 2013, Solar Phys. 287, 343, doi:10.1007/s11207-012-0070-1.

Aschwanden, M.J., Wuelser, J-P., Nitta, N., Leman, J.: 2012a Solar Phys. 281, 101, ADS:2012SoPh..281..101A, doi:10.1007/s11207-012-0092-8.

Aschwanden, M.J., Wuelser, J.-P, Nitta, N.V., Lemen, J.R., DeRosa, M.L., Malanushenko, A.: 2012b, Astrophys. J. 756, 124.

DeRosa, M.L., Schrijver, C.J., Barnes, G., Leka, K.D., Lites, B.W., Aschwanden, M.J., Amari, T., Canou, A., McTiernan, J.M., Rginer, S., Thalmann, J.K., Valori, G., Wheatland, M.S., Wiegelmann, T., Cheung, M.C.M., Conlon, P.A., Fuhrmann, M., Inhester, B., Tadesse, T.: 2009, Astrophys. J. 696, 1780.

Falconer, D. A., Moore, R. L, Gary G.A.: 2002, Astrophys. J. 569, 1016, doi:10.1086/339161

Falconer, D. A., Moore, R. L, Gary G.A.: 2008, Astrophys. J. 689, 1433, doi:10.1086/591045

Falconer, D. A., Moore, R. L, Gary G.A., Adams, M.: 2009, Astrophys. J. Lett. 700, L166. , doi:doi:10.1088/0004-637X/700/2/L166

Gary, G.A.: 1989, Astrophys. J. Suppl. 69, 323.

Gary,G. A., Hu, Q., Lee, J. K.: 2013 Solar Phys. On-line, Paper I, doi:10.1007/s11207-013-0359-8.

Lemen, R.J., Title, A.M., Akin, D.J., Boerner, P.J., Chou, C., Drake, J.J. et al.: 2012, Solar Phys. 275, 17L, ADS:2012SoPh..275...17L, doi:10.1007/s11207-011-9776-8.

Lim, E.-K., Jeong, H., Chae., J., Moon, Y.-J.: 2007, Astrophys. J. 656, 1167.

Lopez Fuentes, M.C., Klimchuk, J.A., Demoulin, P.: 2006, Astrophys. J. 639, 459.

Malanushenko, A., Longcope, D.W., McKenzie, D.E.: 2009, Astrophys. J. 707, 1044.

Malanushenko, A., Yusuf, M.H., Longcope, D.W.: 2011, Astrophys. J. 736, 97.

Marsh, G. E.: 1996, Force-Free Magnetic Fields, World Scientific, NJ.

Mortenson, M.E.: 1997, Geometric Modeling, John Wiley and Sons, NY.

Press, W. H., Flannery, B. P., Teukolsky, S. A., Vetterling, W. T.: 1988 Numerical Recipes: The Art of Scientific Computing, Cambridge, NY.

Scherrer, P.H., Schou, J., Bush, R.I., Kosovichev, A.G., Bogart, R.S., Hoeksema, J.T., Liu, Y., Duvall, T.L., Zhao, J., Title, A.M., Schrijver, C.J., Tarbell, T.D., Tomczyk, S.: 2012, Solar Phys. 275, 207, ADS:2012SoPh..275..207S, doi:10.1007/s11207-011-9834-2.

Tarr, L., Longcope, D., Millhouse, M.: 2013, Astrophys. J. 770, 4, doi:10.1088/0004-637X/770/1/4.

Wiegelmann, T. Inhester, B.: 2010, Astron. Astrophys. 516, 107.

Wiegelmann, T., Yelles, C., Solanki, S.K., Lagg A.: 2010, Astron. Astrophys. 511, A4. 